\section{Gategory}

Metal-Mediated

Synthesis

\section{Key words}

iron

magnesium

alkynes
L. ILIES, T. YOSHIDA, E. NAKAMURA* (THE UNIVERSITY OF TOKYO, JAPAN)

Iron-Catalyzed Chemo- and Stereoselective Hydromagnesiation of Diarylalkynes and Diynes

J. Am. Chem. Soc. 2012, 134, 16951-16954.

\title{
Iron-Catalyzed Hydromagnesiation of Diarylalkynes and Diynes
}

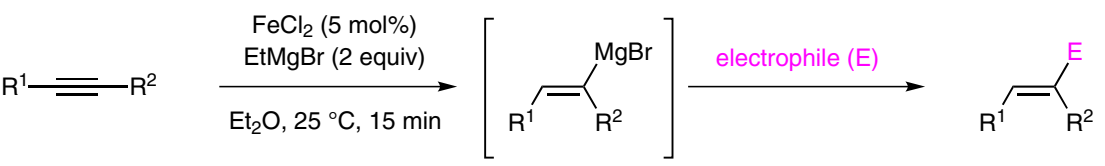

up to $94 \%$ yield E/Z up to $>99: 1$

$\mathrm{R}^{1}=\mathrm{Ph}$, 2-thienyl, 4- $\mathrm{FC}_{6} \mathrm{H}_{4}$, Tol, PMP

$\mathrm{R}^{2}=\mathrm{Ar}$, HetAr, Alk, alkene, and alkyne substituents

$\mathrm{E}=\mathrm{H}_{2} \mathrm{O}, \mathrm{D}_{2} \mathrm{O}$, allyl bromide, DMF, $\mathrm{PhCHO}, \mathrm{Ph}_{2} \mathrm{SiHCl}, \mathrm{Phl}, \mathrm{N}$-ethyl- $\mathrm{N}$-(2-iodobenzyl)ethanamine

Selected examples:

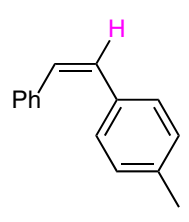

$89 \%$ yield $E / Z=4: 96$

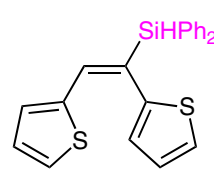

$38 \%$ yield $E / Z>99: 1$<smiles>Oc1ccc(/C=C\c2ccccc2)cc1</smiles>

$75 \%$ yield $E / Z=11: 89$

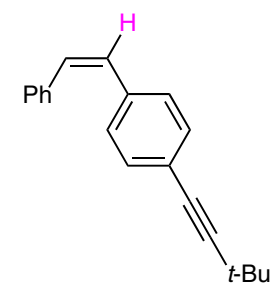

$64 \%$ yield<smiles>OC(/C=C/c1ccccc1)c1ccccc1</smiles><smiles>C(=C(c1ccccc1)c1ccccc1)c1ccccc1</smiles>

$80 \%$ yield

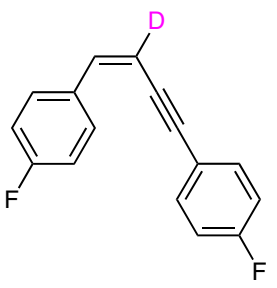

$63 \%$ yield $E / Z=11: 89$
Significance: The authors report a novel ironcatalyzed hydromagnesiation of diarylalkynes in high yield with high stereoselectivity. Furthermore, alkenyl-magnesium compounds can be synthesized from diynes in a chemo-, regio- and stereoselective way.
Comment: The alkenylmagnesium intermediates can further be functionalized in a one-pot sequence. Reactions with allyl bromide, N,N-dimethylformamide and even nickel-catalyzed cross-couplings have been disclosed. The authors suggest a radical mechanism instead of a pure anionic mechanism. 